\title{
HORIZONTAL-BRANCH STARS WITH STRONG HE LINES
}

\author{
Deborah A. Crocker and Robert T. Rood \\ University of Virginia
}

\begin{abstract}
We discuss observations of He lines at $\lambda 4026 \dot{A}$ in two stars in NGC 6752 and three in M 92. M 92 and NGC 6752 are moderate to extreme second parameter clusters. The presence of He lines in stars where diffusion should deplete atmospheric helium may be a clue to the identity of the second parameter. One such candidate which could easily inhibit diffusion is rotation.
\end{abstract}

\section{INTRODUCTION}

Observations of hot HB stars show that diffusion has altered the surface helium abundance (see Greenstein and Sargent 1974). So, in one of the few opportunities we have of directly measuring the He abundance in Pop II stars we are thwarted.

While making spectroscopic observations of blue cluster HB stars to investigate the origin of BHB gaps (see Rood and Crocker 1987) we detected He lines at $\lambda 4026 \AA$ in two stars in NGC 6752 and three in $M$ 92. These lines are moderately strong, indicative of a Pop I helium abundance.

Fig. 1 shows the spectra of the three stars in $M$ 92. The temperature and surface gravity of each star and the equivalent width of the He lines at $\lambda 4026 \dot{A}$ are listed in the figures. Spectra of the stars in NGC 6752 with He lines are shown in Crocker, Rood, and O'Connel1 (1986 = CRO).

\section{IMPLICATIONS}

Heber et al. (1986) have reported measurements of He in NGC 6752 stars. One of the stars they observed was also observed by us (our $4104=$ their 1083). They find reduced abundances (by factors of $1 / 2$ to $3 / 4)$. We feel that neither our spectra, nor theirs are good enough to warrant a detailed analysis though. In addition, there are other observations of hot stars in M 3 and M 13 (Kadla, Gerashchenko, and Yablokova 1985) which show an apparently normal He abundance.

These four clusters (M 3, M 92, M 13 and NGC 6752) are all moderate to extreme second parameter clusters. In addition, $M 92$ and 
NGC 6752 are gap clusters. The helium lines we observe may be related to a gap/second parameter candidate. For instance, rotation, in addition to producing a bluer $\mathrm{HB}$, could induce meridional circulation which could inhibit diffusion. Atmospheric enrichment of helium during core flash would also produce blue stars.

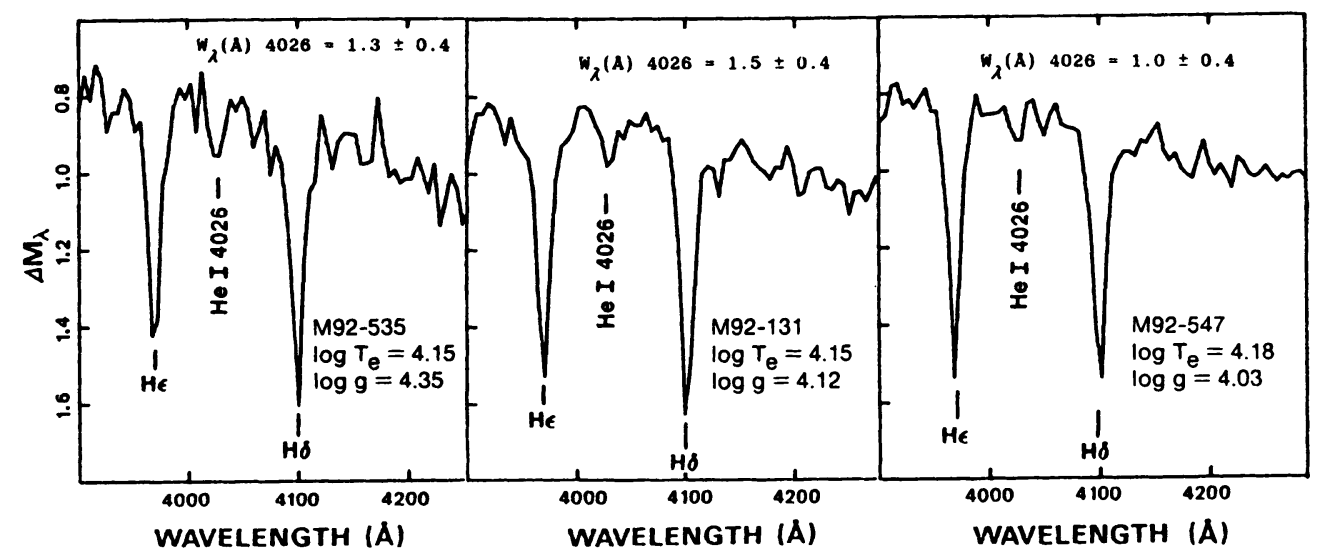

Fig. 1. Spectra of 3 stars in M 92

We do not detect He in observations of a fourth star in M 92 and one in $M 3$ at the same temperature and surface gravity as the stars with He lines. Any explanation for the appearance of the He lines we measure must also account for their absence in otherwise similar stars.

Further discussion on the implications of finding He lines in gap and second parameter clusters may be found in CRO.

\section{AKNOWLEDGEMENTS}

We thank R. Buonanno, F. Fusi Pecci, V. Caloi and their colleagues for providing NGC 6752 data in advance of publication. We also aknowledge use of the observing facilities at CTIO and KPNO.

\section{REFERENCES}

Crocker, D. A., Rood, R. T. and 0'Conne11, R. W. 1986 Astrophys. J. Lett., 309, in press.

Greenstein, J.L. and Sargent, A. I. 1974 Astrophys. J. Supp1., $28,157$.

Heber, U., Kudritski, R. P., Caloi, V., Castellani, V., Danziger, J. and Gilmozzi, R. 1986 Astron. Astrophys., 162, 171.

Kadla, Z. I., Gerashchenko, A. N., and Yablokova, N. V. 1985 Sov. Astron. Lett., 11, 142 .

Rood, R. T. and Crocker, D. A. 1987 in IAU Symposium No. 126. Globular Cluster Systems in Galaxies, J. E. Grindlay and A. G. D. Philip, eds., Reidel, Dordrecht, p. 507. 